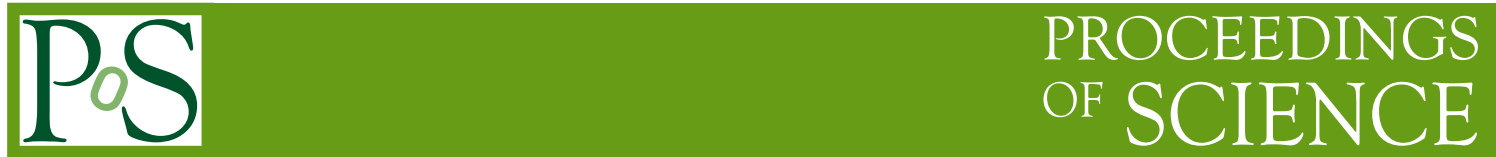

\title{
Organic dust in the interstellar medium
}

\section{Sun Kwok*}

Faculty of Science, The University of Hong Kong, Hong Kong, China

E-mail: sunkwok@hku.hk

The possibility that complex organics are a major component of interstellar dust is discussed. Comparison between astronomical infrared spectra and laboratory spectra of amorphous carbonaceous materials suggests that organics of mixed aromatic-aliphatic structures are widely present in circumstellar, interstellar, and galactic environments. Scenarios for the synthesis of these compounds in the late stages of stellar evolution is presented. We also explore the relationship between interstellar organic dust and complex organics found in the Solar System.

The Life Cycle of Dust in the Universe: Observations, Theory, and Laboratory Experiments - LCDU 2013, 18-22 November 2013

Taipei, Taiwan

\footnotetext{
* Speaker.
} 


\section{Introduction}

Before the 19th century, organic matter was assumed to be associated only with life and was believed to possess a special quality called the "vital force". This belief was based on the fact that biologically related molecules were all derived from living organisms. For example, the amino acid asparagine was derived from asparagus, leucine from cheese, glycine from gelatin, and living yeast was needed for fermentation of sugar into alcohol. However, advances in laboratory chemical techniques have made possible the artificial synthesis of urea from ammonium cyanate in 1823 , amino acid alanine from acetaldehyde, ammonia, and hydrogen cyanide in 1850, and sugars from formaldehyde in 1861 . The ability to synthesize organic matter from inorganic ingredients has led to the demise of the "vital force" concept. We now define organics as the class of molecules and compounds based on the element carbon, but they contain also other elements such as $\mathrm{H}, \mathrm{O}, \mathrm{N}, \mathrm{S}$, and $\mathrm{P}$.

On Earth, organic matter as the result of life is present in the oceans, in the atmosphere, and on the Earth's crust. However, the total amount of biomass ( $~ 2000$ giga tons) pales in comparison to the amount of kerogen $\left(>1.5 \times 10^{7}\right.$ giga tons $)$, a complex organic found in sedimentary rocks [1]. Kerogen is believed to be a precursor of fossil fuels such as oil, coal and natural gas and therefore represents remnants of past life on Earth.

The detection $>100$ gas-phase organic molecules in the interstellar medium through their rotational and vibrational transitions [2] gave us the first hint that organic matter can be naturally synthesized in space. The discovery of the family of unidentified infrared emission (UIE) bands at 3.3, 6.2, 7.7, 8.6 and $11.3 \mu \mathrm{m}$, identified as vibrational bands of aromatic compounds [3], implies that complex organics can be synthesized in the circumstellar and interstellar environments. The detection of the UIE features in distant galaxies [4] suggests that organics were already widely present in the early history of the Universe.

Solid-state particles (commonly referred to in the astronomical literature as dust or grains) have been known to exist in the interstellar medium through the technique of selective extinction for about 100 years. Early suggestions of the chemical structure of interstellar solids included graphite, iron, and ice. The detection of the $10 \mu \mathrm{m}$ silicate feature in evolved stars and in the interstellar medium in the late 1960s led to the realization that minerals such as amorphous silicates are a major component of interstellar solids [5]. Although the existence of organic solids in space has been speculated for some time [6,7], this idea has never been taken seriously by the astronomical community until recently. There is now strong evidence that complex organic matter is widely present in the Solar System, in the circumstellar environment of stars, in interstellar clouds, in the diffuse interstellar medium, and in distant galaxies [8]. In this paper, I will review our current understanding of the chemical structure of organic solids in space.

\section{Synthesis of complex organics in the late stages of stellar evolution}

The UIE features were first discovered in the planetary nebula NGC 7027 [9], and later found to be commonly present in carbon-rich planetary nebulae. Since planetary nebulae are in a short $\left(\sim 10^{4} \mathrm{yr}\right.$ ) phase of stellar evolution after the end of the asymptotic giant branch (AGB) evolution [10], the detection of UIE bands in planetary nebulae but not in AGB stars provides strong evidence 
that organic synthesis can take place in the circumstellar environment over short time scales. The best objects to study the early synthesis of stellar organics are proto-planetary nebulae, objects in transition between the AGB and planetary nebulae stages [11]. Infrared spectroscopic observations of proto-planetary nebulae show that they not only possess the aromatic emission features, but also aliphatic features at 3.38, 3.42, 3.46, 3.49, and 3.51 $\mu \mathrm{m}$ due to $\mathrm{C}-\mathrm{H}$ stretch of methyl and methylene groups attached to aromatic rings [12]. In addition, $\mathrm{C}-\mathrm{H}$ out-of-plane bending modes at $12.1,12.4$, and $13.3 \mu \mathrm{m}$ due to aromatic rings with 2,3 , or 4 exposed edges are also detected in proto-planetary nebulae [13]. This suggests that the number of rings in each aromatic network group may be small. Most interestingly, strong broad emission plateaus around 8 and $12 \mu \mathrm{m}$ are commonly seen as well. These plateau emission features are identified as a superposition of inplane and out-of-plane bending modes of a variety of aliphatic side groups [14]. Consequently, an extensive network of aliphatic groups may be present.

\section{Carrier of the unidentified infrared emission bands}

The UIE bands are characterized by a group of aromatic features at 3.3, 6.2, 7.7, 8.6, and 11.3 $\mu \mathrm{m}$, aliphatic features at 3.4 and $6.9 \mu \mathrm{m}$, emission features of uncertain assignments at 15.8, 16.4, $17.4,17.8$, and $18.9 \mu \mathrm{m}$, and broad plateau features at 8,12 , and $17 \mu \mathrm{m}$. A successful model has to account for all these spectral characteristics.

The UIE bands are widely observed in a variety of astrophysical environments. These include planetary and proto-planetary nebulae, star formation regions, HII regions, reflection nebulae, novae, diffuse interstellar medium, and external galaxies. In addition to emission, the 3.4 and 6.9 $\mu \mathrm{m}$ aliphatic features are seen in absorption against the infrared continuum of the galactic center $[15,16]$. Since abundance can easily be derived from the strengths of absorption features, it is estimated that the amount of carbon tied up in aliphatic compounds represent $15-30 \%$ of the total interstellar carbon [17]. Akari observations of M82 have shown that the strength of the 3.4 $\mu \mathrm{m}$ feature increases from the disk to the halo of the galaxy, suggesting that aliphatic organics are distributed throughout the volume of galaxies [18].

In the last 25 years, the hypothesis that the UIE features are due to polycyclic aromatic hydrocarbon molecules $[19,20]$ has been very popular. The essence of the PAH hypothesis is summarized by Tielens: "These features are (almost) universally attributed to the IR fluorescence of far-ultraviolet (FUV)-pumped polycyclic aromatic hydrocarbon (PAH) molecules, containing 50 $\mathrm{C}$ atoms" [21]. The strongest argument for the PAH hypothesis is that single-photon excitation of PAH molecules can account for the $12 \mu \mathrm{m}$ excess emission observed in cirrus clouds in the diffuse interstellar medium $[22,23]$. However, the PAH hypothesis suffers from the following problems:

- PAH molecules have well-defined sharp features but the UIE features are broad;

- PAHs are primarily excited by UV, with little absorption in the visible, but UIE features are seen in proto-planetary nebulae and reflection nebulae, objects with very little UV background radiation;

- The shapes and peak wavelengths of UIE features are independent of temperature of the exciting stars; 


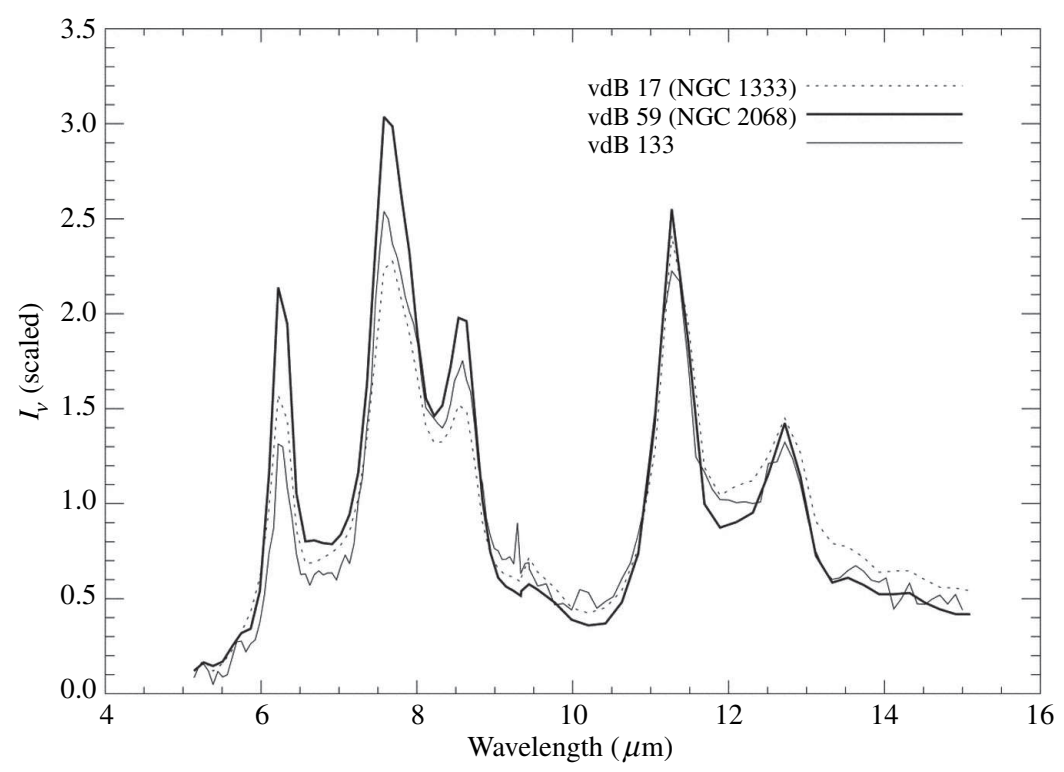

Figure 1: ISO spectra of three reflection nebulae showing that the UIE band profiles and peak weavlengths are independent of the central star temperatures. The reflection nebulae NGC 1333, NGC 2068, and vdB 133 have central stars of temperatures 11000,19000 and $6800 \mathrm{~K}$, respectively (from reference [29]).

- The strong and narrow PAH gas phase features in the UV are not seen in interstellar extinction curves [24, 25];

- No specific PAH molecule has been detected in spite of the fact that the vibrational and rotational frequencies of PAH molecules are well known;

- No laboratory PAH spectrum has been able to reproduce the astronomical UIE spectrum w.r.t. either band positions or relative intensities [26, 27, 28];

- In order to fit the astronomical observations, the PAH model has to appeal to a mixture of PAH of different sizes, structures (compact, linear, branched) and ionization states, as well as artificial broad intrinsic line profiles.

Figure 1 shows that the UIE features have consistent profiles and peak wavelengths in spite of the fact that the nebulae are heated by stars of very different temperatures. Since the PAH model requires PAH ions for visible absorption, it is very unlikely that the consistent astronomical spectra are produced by the exact same mix of diverse PAH molecules in these three nebulae.

\section{Organic solids as carrier of the UIE bands}

In order to identify possible carriers of the UIE bands, it would be useful to see what carbonaceous products can naturally exist in the interstellar medium. By introducing $\mathrm{H}$ into graphite $\left(s p^{2}\right)$ and diamond $\left(s p^{3}\right)$, a variety of amorphous $\mathrm{C}-\mathrm{H}$ alloys can be created $[30,31,32,33]$. Different geometric structures with long- and short-range can be created by varying the aromatic to aliphatic ratios. A schematic of possible structures of amorphous carbonaceous solids is shown in Figure 2. 


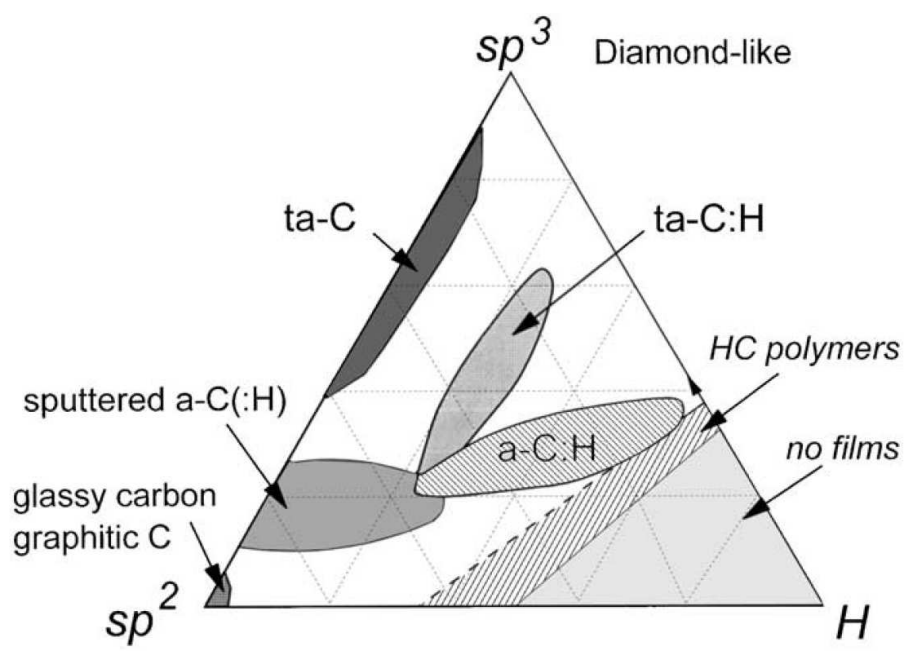

Figure 2: Phase diagram of amorphous carbon based on pure $\mathrm{C}$ and $\mathrm{H}$ compounds (from reference [34]).

The lower left corner of the triangle represents graphite, the top corner represents diamonds, PAHs are on the bottom edge, and various forms of amorphous hydrogenated carbon can exist in the middle. The infrared spectra of these amorphous carbonaceous materials [35] resemble the astronomical UIE bands seen in planetary nebulae and proto-planetary nebulae (Figure 3). Since these amorphous carbonaceous solids have absorption bands in the visible, they can be easily excited by visible light from stars.

Organic particles of such structures are natural products of combustion. The first nucleation products for soot particles formed in flames have structures consisting of islands of aromatic rings linked by chains. Identified sidegroups in soot include methyl $\left(-\mathrm{CH}_{3}\right)$, methylene $\left(-\mathrm{CH}_{2}\right)$, carbonyl $(\mathrm{C}=\mathrm{O})$, aldehydic $(-\mathrm{HCO})$, phenolic $(-\mathrm{OH})$, and amino $\left(-\mathrm{NH}_{2}\right)$ groups [44]. Similar combustion processes may be at work in carbon-rich circumstellar envelopes.

By the early 1980s, it was known that carbon clusters can be produced by laser vaporization of graphite followed by supersonic expansion into an inert gas. The employment of this technique has led to the discovery of fullerene, a new form of carbon [36]. Since then, various techniques based on laser pyrolysis of gas-phase hydrocarbons followed by condensation have been used to create laboratory counterparts of cosmic organic dust [37]. These include the quenching of plasma of 4-torr methane [38], hydrocarbon flame or arc-discharge in a neutral of hydrogenated atmosphere $[39,40]$, laser ablation of graphite in a hydrogen atmosphere [41, 42], infrared laser pyrolysis of gas phase molecules $\left(\mathrm{C}_{2} \mathrm{H}_{4}, \mathrm{C}_{4} \mathrm{H}_{6}\right)$ [43], photolysis of methane at low temperatures [16], and flame combustion of $\mathrm{C}_{2} \mathrm{H}_{2}, \mathrm{C}_{2} \mathrm{H}_{4}, \mathrm{C}_{3} \mathrm{H}_{6}$ mixed with $\mathrm{O}_{2}$, forming soot [44].

In addition to soot, there are also other natural products such as coal and kerogen that have similar amorphous mixed aromatic/aliphatic properties [45, 46]. The infrared spectra of petroleum and asphaltenes also show spectral features similar to astronomical UIE bands [47, 48].

Although they are part of the family of UIE features, the exact vibrational mode assignments of the features at $16.4,17.4,18.9 \mu \mathrm{m}$ are not clear. It is possible that the 17.4 and $18.9 \mu \mathrm{m}$ features are due to, or receive contributions from, the $\mathrm{C}_{60}$ molecule. The $16-20 \mu \mathrm{m}$ region is generally associated with twisting, bending, and wagging modes of the aromatic carbon skeleton [49]. Some 

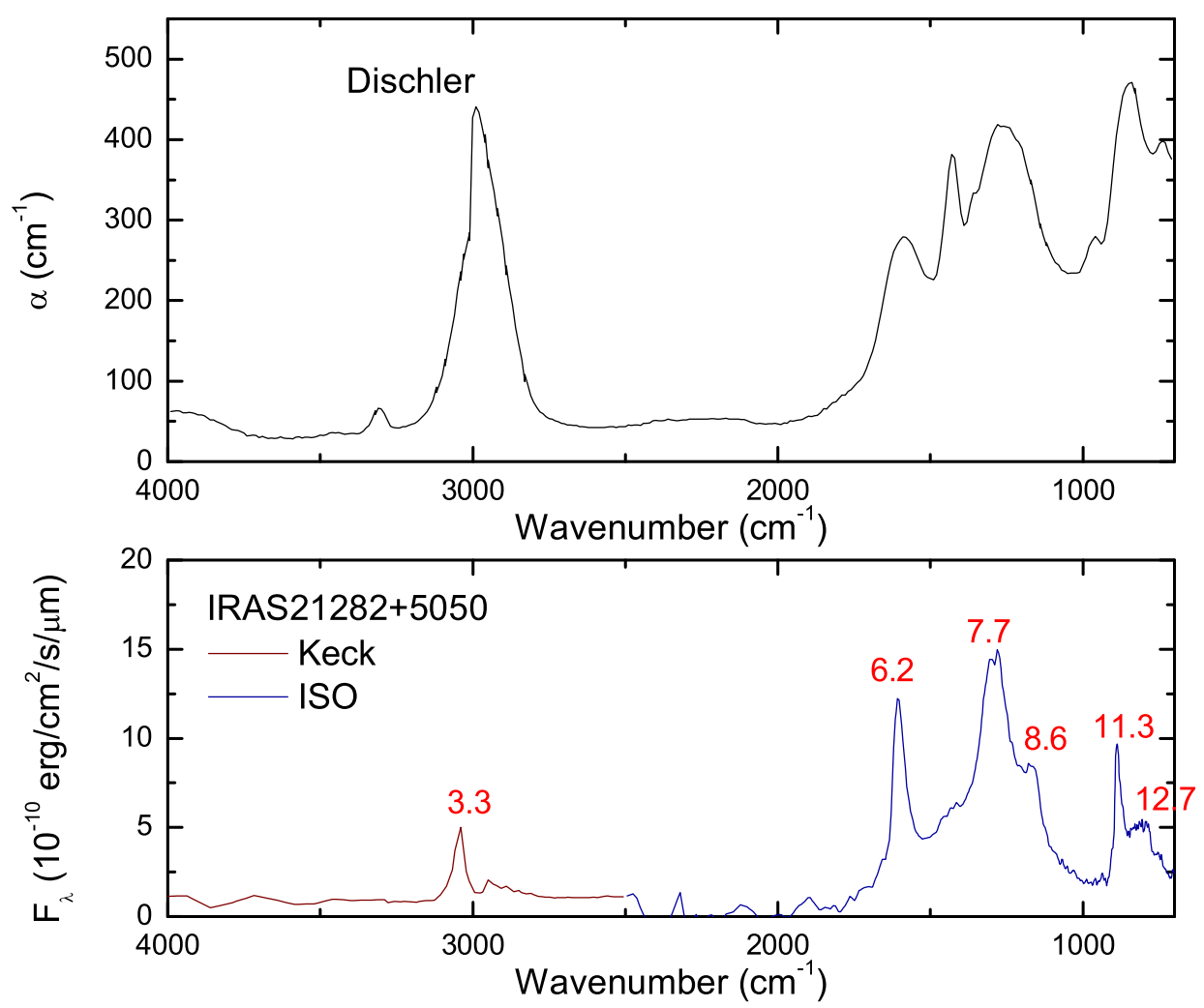

Figure 3: Laboratory infrared spectra of hydrogenated amorphous carbon (top, from reference [35]) compared to the astronomical spectrum of the planetary nebula IRAS 21282+5050 (bottom panel). The UIE bands are labeled by their wavelengths in $\mu \mathrm{m}$.

of these features can be seen in incompletely hydrogenated carbon nanoparticles with sizes of $10^{2}-10^{4} \mathrm{C}$ atoms [50]. Nanoparticles with thousands of $\mathrm{C}$ atoms are unlikely to be heated by the single-photon process. An alternative source of heating is chemical heating [51].

\section{The MAON model}

The detection of aliphatic features implies that the carrier of the UIE bands cannot be a pure aromatic molecule like PAH but must incorporate an aliphatic component. This can be done by inserting aliphatic side groups to a large cluster of aromatic rings [14, 52]. A more extreme model would have the aromatic and aliphatic units in a completely disorganized structure. Figure 4 shows an example of the mixed aromatic/aliphatic organic nanoparticles (MAON) model where small units of aromatic rings are linked by aliphatic chains of random lengths oriented at random directions in a 3-D structure $[53,54]$. Since heavy elements are part of the stellar outflows, it is quite possible that such structures will contain impurities such as $\mathrm{O}, \mathrm{S}$, and $\mathrm{N}$ and these are illustrated in Figure 4.

\section{Fullerene and MAON}

The discovery of fullerene $\left(\mathrm{C}_{60}\right)$ in the planetary nebula Tc-1 [55] has generated interests in 


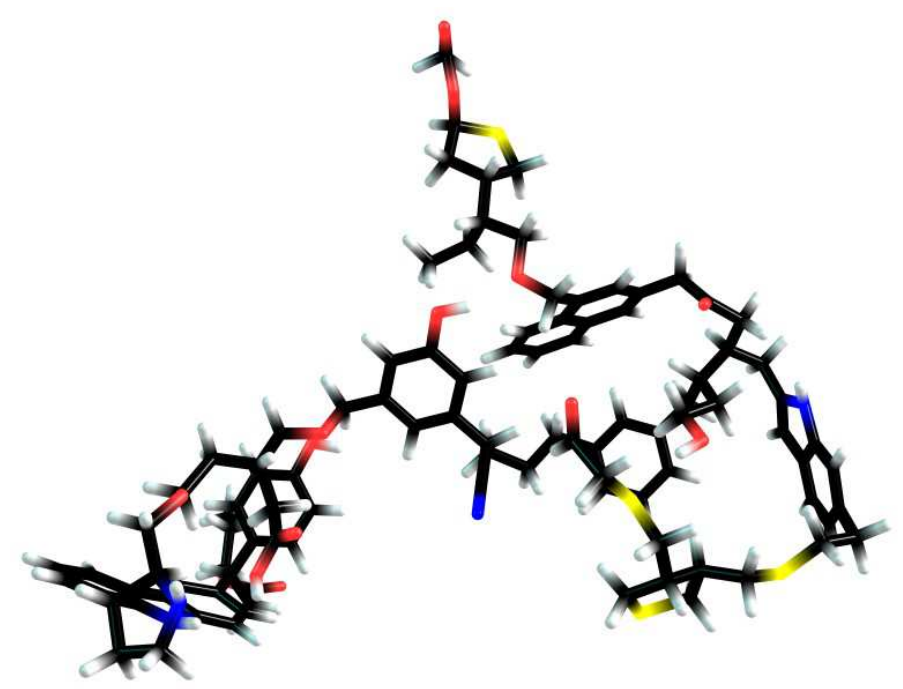

Figure 4: A 3-D illustration of a possible partial structure of a MAON particle. Carbon atoms are represented in black, hydrogen in light grey, sulphur in yellow, oxygen in red, and nitrogen in blue. There are $101 \mathrm{C}$, $120 \mathrm{H}, 14 \mathrm{O}, 4 \mathrm{~N}$, and $4 \mathrm{~S}$ atoms in this example. The number of heavy elements have been intentionally exaggerated for the purpose of illustration.

possible links between $\mathrm{C}_{60}$ and the UIE phenomenon. Although Tc-1 has no UIE features, it does show the 8 and $12 \mu \mathrm{m}$ plateaus (Fig. 5). The proto-planetary nebula IRAS $01005+7910$, however, show $\mathrm{C}_{60}$ features, UIE bands, as well as the 8,12 , and $17 \mu \mathrm{m}$ emission plateau features [56]. The association between $\mathrm{C}_{60}$ and the 8 and $12 \mu \mathrm{m}$ plateau features $[57,58]$ suggests that amorphous carbonaceous solids such as MAONs could be precursors of fullerenes [59, 60].

\section{Relationship between stellar, interstellar, and Solar System organics}

Although it was commonly believed that Solar System objects were made of minerals, metals, and ices, we now know that meteorites, asteroids, comets, planetary satellites, and interplanetary dust particles all contain organic materials. In the soluble component of carbonaceous chondrites, almost all biologically relevant organic compounds can be found [62]. The insoluble organic matter (IOM) of carbonaceous chondrites is composed of highly substituted single ring aromatics, substituted furan/pyran functional groups, highly branched oxygenated aliphatics and carbonyl groups [63]. The $3.4 \mu \mathrm{m}$ feature is found in meteorites [64, 65], interplanetary dust particles [66], comets [67], and in the haze of Titan [68] and Saturn [69]. The total amount of complex hydrocarbons in the lakes and dunes of Titan exceed the total oil and gas reserves on Earth [70].

The organic component in Solar System objects is believed to have formed during the early days of the Solar System. Given the spectral similarity between stellar and Solar System organics, is it possible that there is a link between stellar and Solar System organics [71, 72]? It has been suggested that the $3.4 \mu \mathrm{m}$ feature exhibited in comets is the result of warming of interstellar organic matter embedded in comets during the early stages of the Solar System [73]. Because the timescales of chemical synthesis in circumstellar envelopes are constrained by the short dynamical and evolutionary timescales, stellar outflows are the only sources which we can directly witness the 


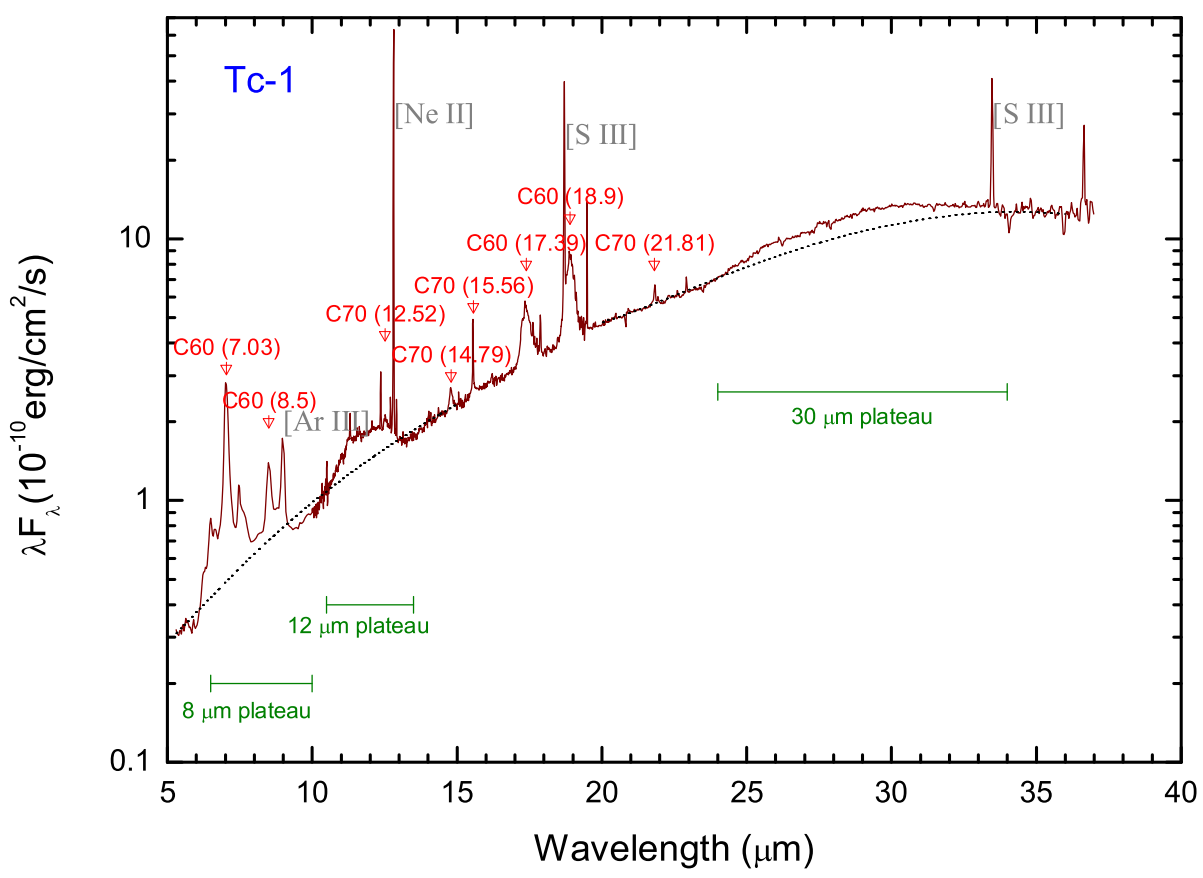

Figure 5: The Spitzer IRS spectrum of planetary nebula Tc-1 shows $\mathrm{C}_{60}$ and $\mathrm{C}_{70}$ emission bands (in red) as well as broad emission plateau features at 8,12 , and $30 \mu \mathrm{m}$ (in green). The narrow lines are atomic lines (in grey). No UIE bands are seen in this object.

formation of complex organics. We know that evolved stars are able to distribute stellar minerals to the Solar System in the form of pre-solar grains [74]. It has also been noted that some IOM carries isotopic signatures suggesting an interstellar origin $[75,76]$. The possibility that the early Solar System has been chemically enriched by stellar ejecta cannot be ruled out.

\section{Conclusions}

Although the first interstellar molecule was found in the 1930s, the idea that molecular matter is a major component of the Universe was not firmly established until the 1970s. Carbon, being highly abundant and versatile, must play a major role in the chemistry of interstellar matter. Amorphous hydrocarbons of mixed aromatic and aliphatic structures can be a natural product of interstellar chemistry.

From infrared spectroscopic observations from space, we now have evidence that complex organic compounds are everywhere in the Universe - from Solar System to the interstellar medium to galaxies. The detection of UIE bands in galaxies with $z \sim 2$ suggests that complex organics are already present in the early history of the Universe. The observed properties of the UIE features are consistent with the carrier being mixed aromatic/aliphatic organic nanoparticles (MAON), or some other similar forms of amorphous carbonaceous solid. From spectral observations of objects 
in the late stages of stellar evolution, we learn that chemical evolution leading to complex organic compounds can take place over only a few thousand years in the circumstellar environment. Given the widespread presence and complexity of Solar System organics, the possible roles of evolved stars in the enrichment of the Galaxy and the Solar System need to be further studied.

Acknowledgments: I would like to thank my colleagues Yong Zhang and SeyedAbdolreza Sadjadi for helpful discussions. This work was partially supported by the Research Grants Council of the Hong Kong Special Administrative Region, China (project no. HKU 7031/10P).

\section{References}

[1] Falkowski, P., et al. 2000, Science, 290, 291

[2] Kwok, S. 2007, Physics and Chemistry of the Interstellar Medium, University Science Books

[3] Duley, W. W., and Williams, D. A. 1981, Mon. Not. Roy. Astr. Soc., 196, 269

[4] Smith, J. D. T. et al. 2007, Astrophys. J., 656, 770

[5] Woolf, N. J., \& Ney, E. P. 1969, Astrophys. J., 155, L181

[6] Hoyle, F., and Wickramasinghe, N. C. 1977, Nature, 268, 610

[7] Knacke, R. F. 1977, Nature, 269, 132

[8] Kwok, S. 2011, Organic Matter in the Universe, Wiley

[9] Russell, R. W., Soifer, B. T., and Willner, S. P. 1977, Astrophys. J., 217, L149

[10] Kwok, S. 2000, Origin and Evolution of Planetary Nebulae, Cambridge University Press

[11] Kwok, S. 1993, Ann. Rev. Astron. Astrophys., 31, 63

[12] Hrivnak, B. J., Geballe, T. R., \& Kwok, S. 2007, Astrophys. J., 662, 1059

[13] Kwok, S., Volk, K., and Hrivnak, B. J. 1999, Astron. Astrophys., 350, L35

[14] Kwok, S., Volk, K., and Bernath, P. 2001, Astrophys. J., 554, L87

[15] Chiar, J. E., Adamson, A. J., Pendleton, Y. J., Whittet, D. C. B., Caldwell, D. A., \& Gibb, E. L. 2002, Astrophys. J., 570, 198

[16] Dartois, E., Muñoz Caro, G. M., Deboffle, D., \& d'Hendecourt, L. 2004, Astron. Astrophys., 423, L33

[17] Dartois, E. 2011, in PAHs and the Universe, eds. Joblin, \& Tielens (EAS Publications Series), 381

[18] Yamagishi, M., Kaneda, H., Ishihara, D., Kondo, T., Onaka, T., Suzuki, T., \& Minh, Y. C. 2012, Astron. Astrophys., 541, 10

[19] Allamandola, L. J., Tielens, A. G. G. M., and Barker, J. R. 1989, Astrophys. J. Suppl., 71, 733

[20] Puget, J. L., and Léger, A. 1989, Ann. Rev. Astron. Astrophys., 27, 161

[21] Tielens, A. G. G. M. 2008, Ann. Rev. Astron. Astrophys., 46, 289

[22] Sellgren, K. 1984, Astrophys. J., 277, 623

[23] Sellgren, K. 2001, Spectrochimica Acta, 57, 627

[24] Gredel, R., Carpentier, Y., Rouillé, G., Steglich, M., Huisken, F., \& Henning, T. 2011, Astron. Astrophys., 530, 26 
[25] Salama, F., Galazutdinov, G. A., Krelowski, J., Biennier, L., Beletsky, Y., \& Song, I.-O. 2011, Astrophys. J., 728, 154

[26] Cook, D. J., Schlemmer, S., Balucani, N., Wagner, D. R., Steiner, B., \& Saykally, R. J. 1996, Nature, 380, 227

[27] Cook, D. J., \& Saykally, R. J. 1998, Astrophys. J., 493, 793

[28] Wagner, D. R., Kim, H., \& Saykally, R. J. 2000, Astrophys. J., 545, 854

[29] Uchida, K. I., Sellgren, K., Werner, M. W., \& Houdashelt, M. L. 2000, Astrophys. J., 530, 817

[30] Jones, A. P. 2012, Astron. Astrophys., 540, 2

[31] Jones, A. P. 2012, Astron. Astrophys., 540, 1

[32] Jones, A. P. 2012, Astron. Astrophys., 545, 3

[33] Jones, A. P., Fanciullo, L., Köhler, M., Verstraete, L., Guillet, V., Bocchio, M., \& Ysard, N. 2013, Astron. Astrophys., 558, 62

[34] Robertson, J. 2002, Material Science and Engineering R, 37, 129

[35] Dischler, B., Bubenzer, A., \& Koidl, P. 1983, Solid State Communications, 48, 105

[36] Kroto, H. W., Heath, J. R., Obrien, S. C., Curl, R. F., \& Smalley, R. E. 1985, Nature, 318, 162

[37] Jäger, C., Huisken, F., Mutschke, H., Jansa, I. L., \& Henning, T. H. 2009, Astrophys. J., 696, 706

[38] Sakata, A., Wada, S., Onaka, T., \& Tokunaga, A. T. 1987, Astrophys. J., 320, L63

[39] Colangeli, L., et al. 2003, Astron. Astrophys. Rev., 11, 97

[40] Mennella, V., Baratta, G. A., Esposito, A., Ferini, G., \& Pendleton, Y. J. 2003, Astrophys. J., 587, 727

[41] Scott, A., \& Duley, W. W. 1996, Astrophys. J., 472, L123

[42] Mennella, V., Brucato, J. R., Colangeli, L., \& Palumbo, P. 1999, Astrophys. J., 524, L71

[43] Herlin, N., Bohn, I., Reynaud, C., Cauchetier, M., Galvez, A., \& Rouzaud, J.-N. 1998, Astron. Astrophys., 330, 1127

[44] Pino, T., et al. 2008, Astron. Astrophys., 490, 665

[45] Guillois, O., Nenner, I., Papoular, R., \& Reynaud, C. 1996, Astrophys. J., 464, 810

[46] Papoular, R. 2001, Astron. Astrophys., 378, 597

[47] Cataldo, F., Keheyan, Y., \& Heymann, D. 2002, International Journal of Astrobiology, 1, 79

[48] Cataldo, F., Garcia-Hernádez, D.A., \& Manchado, A. 2013, Mon. Not. Roy. Astr. Soc., 429, 3025

[49] Cataldo, F., Angelini, G., Aníbal García-Hernández, D., \& Manchado, A. 2013, Spectrochimica Acta Part A: Molecular and Biomolecular Spectroscopy, 111, 68

[50] Hu, A., \& Duley, W. W. 2008, Astrophys. J., 672, L81

[51] Duley, W.W., \& Williams, D.A. 2011, Astrophys. J., 737, L44

[52] Pendleton, Y. J., \& Allamandola, L. J. 2002, Astrophys. J. Suppl., 138, 75

[53] Kwok, S. \& Zhang, Y. 2011, Nature, 479, 80

[54] Kwok, S., \& Zhang, Y. 2013, Astrophys. J., 771, 5 
[55] Cami, J., Bernard-Salas, J., Peeters, E., \& Malek, S. E. 2010, Science, 329, 1180

[56] Zhang, Y., Kwok, S., \& Hrivnak, B. J. 2010, Astrophys. J., 725, 990

[57] Zhang, Y., \& Kwok, S. 2013, Earth, Planets, and Space, 65, 1069

[58] Otsuka, M., Kemper, F., Cami, J., Peeters, E., \& Bernard-Salas, J. 2014, Mon. Not. Roy. Astron. Soc. , 437, 2577

[59] García-Hernández, D. A., et al. 2012, Astrophys. J., 760, 107

[60] Bernard-Salas, J., Cami, J., Peeters, E., Jones, A. P., Micelotta, E. R., \& Groenewegen, M. A. T. 2012, Astrophys. J., 757, 41

[61] Micelotta, E., Jones, A.P., Peeters, E., Bernard-Salas, J., \& Fanchini, G. 2012, Astrophys. J., 761,35

[62] Schmitt-Kopplin, P., et al. 2010, Proc. Nat. Acad. Sci., 107, 2763

[63] Cody, G. D., Heying, E., Alexander, C.M.O., Nittler, L.R., Kilcoyne, A.L.D., Sandford, S.A., Stroud, R.M. 2011, Proc. Nat. Acad. Sci., 108, 19171

[64] Cronin, J.R., \& Pizzarello, S., 1990, Geochimica et Cosmochimica Acta, 54, 2859.

[65] Ehrenfreund, P., Robert, F., D’Hendecourt, L., \& Behar, F. 1991, Astron. Astrophys., 252, 712

[66] Flynn, G. J., Keller, L. P., Feser, M., Wirick, S., \& Jacobsen, C. 2003, Geochimica et Cosmochimica Acta, 67, 4791

[67] Keller, L. P., et al. 2006, Science, 314, 1728

[68] Kim, S. J., et al. 2011, Planetary and Space Science, 59, 699

[69] Kim, S.J., Sim, C.K., Lee, D.W., Courtin, R., Moses, J.I., Minh, Y.C. 2012, Planetary and Space Science 65, 122

[70] Lorenz, R. D., et al. 2008, Geo. Res. Letters, 35, 2206

[71] Kwok, S. 2004, Nature, 430, 985

[72] Kwok, S. 2013, Stardust: the cosmic seeds of life, Springer

[73] Mennella, V. 2010, Astrophys. J., 718, 867

[74] Zinner, E. 1998, Ann. Rev. Earth \& Planetary Sci., 26, 147

[75] Kerridge, J. F. 1999, Space Sci. Rev., 90, 275

[76] Alexander, C.M.O’D., Cody, G.D., Fogel, M., \& Yabuta, H. 2008, in IAU Symp. 251: Organic Matter in Space, eds. S. Kwok \& S. Sandford, CUP, pp. 293 